\title{
Lower Limbic Metabotropic Glutamate Receptor 5 Availability in Alcohol Dependence
}

\author{
Gil Leurquin-Sterk ${ }^{1}$, Jenny Ceccarini ${ }^{1}$, Cleo L. Crunelle ${ }^{2,3}$, Bart de Laat ${ }^{1,4}$, Jef Verbeek ${ }^{5}$, Stephanie Deman ${ }^{6}$, Hugo Neels $^{2}$, \\ Guy Bormans ${ }^{7}$, Hendrik Peuskens ${ }^{8}$, and Koen Van Laere ${ }^{1,4}$ \\ ${ }^{I}$ Division of Nuclear Medicine and Molecular Imaging, Department of Imaging and Pathology, KU Leuven, Leuven, Belgium; \\ ${ }^{2}$ Toxicological Center, University of Antwerp, Wilrijk, Belgium; ${ }^{3}$ Department of Psychiatry, University Hospital Brussels, Brussels, \\ Belgium; ${ }^{4}$ MoSAIC: Molecular Small Animal Imaging Center, KU Leuven, Leuven, Belgium; ${ }^{5}$ Division of Gastroenterology and \\ Hepatology, Department of Internal Medicine, Maastricht University Medical Center, Maastricht, The Netherlands; ${ }^{6}$ Genomics Core, \\ UZ Leuven, and Department of Human Genetics, KU Leuven, Leuven, Belgium; ${ }^{7}$ Laboratory for Radiopharmacy, KU Leuven, Leuven, \\ Belgium; and ${ }^{8}$ University Psychiatric Center, KU Leuven, Kortenberg, and Kliniek Broeders Alexianen, Tienen, Belgium
}

\begin{abstract}
Animal studies suggest an important role for the metabotropic glutamate receptor subtype 5 (mGlu5) in the pathophysiology of alcohol dependence, but direct human evidence is lacking. The goal of this study was to investigate cerebral mGlu5 availability in alcohol-dependent subjects versus controls using ${ }^{18} \mathrm{~F}-3-$ fluoro-5[(pyridin-3-yl)ethynyl]benzonitrile ( ${ }^{18}$ F-FPEB) PET. Methods: Dynamic $90-\min { }^{18} \mathrm{~F}$-FPEB scans combined with arterial blood sampling were acquired for 16 recently abstinent alcohol-dependent subjects and 32 age-matched controls. Regional mGlu5 availability was quantified by the ${ }^{18} \mathrm{~F}$-FPEB total distribution volume using both a voxel-by-voxel and a volume-of-interest analysis with partial-volume effect correction. Alcohol consumption within the last 3 mo was assessed by questionnaires and by hair ethyl glucuronide analysis. Craving was assessed using the Desire for Alcohol Questionnaire. Results: mGlu5 availability was lower in mainly limbic regions of alcohol-dependent subjects than in controls $(P<0.05$, familywise error-corrected), ranging from $14 \%$ in the posterior cingulate cortex to $36 \%$ in the caudate nucleus. Lower mGlu5 availability was associated with higher hair ethyl glucuronide levels for most regions and was related to a lower level of craving specifically in the middle frontal gyrus, cingulate cortex, and inferolateral temporal lobe. Conclusion: These findings provide human in vivo evidence that limbic mGlu5 has a role in the pathophysiology of alcohol dependence, possibly involved in a compensatory mechanism helping to reduce craving during abstinence.
\end{abstract}

Key Words: PET; mGlu5; alcohol dependence; craving

J Nucl Med 2018; 59:682-690

DOI: 10.2967/jnumed.117.199422

I

2009, Peter W. Kalivas proposed "the glutamate homeostasis hypothesis of addiction." According to it, the transition from controlled drug use to dependence involves long-lasting glutamatergic neuroadaptations in corticolimbic areas (1). Among others, impaired metabotropic glutamate receptor subtype 5 (mGlu5)-dependent signaling is hypothesized to represent a key component of these adaptations. mGlu5 is a

Received Jul. 18, 2017; revision accepted Oct. 30, 2017.

For correspondence or reprints contact: Jenny Ceccarini, Division of Nuclear Medicine and Molecular Imaging, UZ Leuven, Herestraat 49, 3000 Leuven, Belgium.

E-mail: jenny.ceccarini@uzleuven.be

Published online Jan. 18, 2018.

COPYRIGHT (C 2018 by the Society of Nuclear Medicine and Molecular Imaging. widely distributed G11/Gq-protein-coupled receptor of the human brain, with the highest expression in corticolimbic areas (2). Mainly located on postsynaptic terminals, mGlu5 is integral to synaptic plasticity processes, partly via its functional positive coupling with the $N$-methyl-D-aspartate receptor (3). Kalivas' theory posits that chronic drug administration results in a mGlu5 downregulation representing a compensatory adaptation helping to reduce compulsive drugseeking. Hence, mGlu5 has been proposed as a promising target for the treatment of several types of substance dependence (4).

Alcohol dependence is a major health concern associated with high societal costs worldwide (5) and with high relapse rates despite available therapies. Therefore, understanding the neural mechanisms that mediate the transition from controlled use to dependence in humans remains a priority of alcohol research.

To date, few preclinical studies have investigated the effect of chronic alcohol administration on mGlu5 density, revealing either a mGlu5 hypoexpression in the hippocampus (6), an overexpression in the amygdala (7), or no alterations (8) or overexpression (9) in the ventral striatum. In contrast, extensive research showed that blocking of mGlu5 signaling by genetic or pharmacologic interventions reduces alcohol-rewarding properties (10-14) and the severity of withdrawal and relapse behaviors after alcohol deprivation in rodents $(13,15-19)$.

In the last $10 \mathrm{y}$, the highly selective and potent mGlu5 PET radioligand ${ }^{18} \mathrm{~F}-3$-fluoro-5-[(pyridin-3-yl)ethynyl]benzonitrile $\quad\left({ }^{18} \mathrm{~F}\right.$ FPEB) has allowed quantification of mGlu5 availability (20), thereby providing a unique opportunity to examine how mGlu5 may adapt across the different stages of alcohol dependence. Noteworthy, ${ }^{18} \mathrm{~F}$ FPEB PET has shown that limbic mGlu5 positively relates to novelty seeking (21), a heritable temperament trait associated with increased susceptibility to drug dependence (22). Moreover, genetic studies have demonstrated that individuals with specific mGlu5 polymorphisms are at higher risk for developing alcohol dependence $(23,24)$. However, the in vivo status of mGlu5 availability in alcohol-dependent subjects and its possible relation to clinical outcome are unknown.

Here, we used ${ }^{18}$ F-FPEB PET to compare mGlu5 availability between recently abstinent alcohol-dependent subjects and controls. Considering the largely shared neurobehavioral deficits observed among various types of drug dependence (25) and the known hyperglutamatergic state associated with alcohol withdrawal (26), we hypothesized that short-term alcohol abstinence would also be associated with lower cerebral mGlu5 availability. Additionally, we addressed whether a potentially lower mGlu5 availability in alcohol-dependent subjects is related to the amount of prior alcohol consumption and to craving. 


\section{MATERIALS AND METHODS}

\section{Participants}

Subjects with a diagnosis of alcohol dependence, according to the Diagnostic and Statistical Manual of Mental Disorders, 4th edition, were recruited by a board-certified psychiatrist specializing in addiction at the Psychiatric Hospital Alexianen Tienen and the Psychiatry Department of the University Hospital Leuven (Belgium). All subjects were included within the first 2 wk of supervised abstinence. Exclusion criteria were any of the following: other psychiatric diseases (including those in a firstdegree relative); substance use disorders other than nicotine dependence; chronic use of benzodiazepines; and abnormal findings on physical examination, blood tests (with a tolerance for liver tests of $\leq 2$ times the reference limit), urine toxicology tests (MULTI Urine DrugControl cassette; ulti med), or structural MRI. According to the local clinical procedure, 6 subjects temporarily received clorazepate (mean cumulative dose, $100 \mathrm{mg}$; range, 50-225 mg) to relieve withdrawal symptoms.
Healthy controls were recruited through local advertisement and underwent a full medical and mental assessment at study intake. The sample for the current study represents a subsample of a previous study (21) and was randomly selected on the basis of age by a person masked to both study protocols in order to obtain 2 age-matched controls for each alcohol-dependent subject (Table 1). Subjects reporting alcohol consumption at a rate of more than 7 units/wk ( 1 unit $=10 \mathrm{~g}$ of alcohol) or regular binge drinking ( $\geq 5$ units per occasion) were excluded. Temperament traits were assessed using the 240-item Cloninger Temperament and Character Inventory (27).

The study protocol was approved by the Ethics Committee of the University Hospital Leuven. All participants signed an informed consent form.

\section{Assessment of Alcohol Use and Craving}

Alcohol consumption in the prior 3 mo was determined subjectively by the TimeLine Followback Method (28). Excessive alcohol use was further assessed using the Alcohol Use Disorders Identification Test

TABLE 1

Overview of Participant Characteristics

\begin{tabular}{|c|c|c|c|}
\hline Characteristic & Alcohol-dependent subjects $(n=16)$ & Controls $(n=32)$ & $P$ \\
\hline Female/male $(n)$ & $3 / 13$ & $14 / 18$ & 0.088 \\
\hline Age $(y)$ & $46 \pm 8$ & $45 \pm 13$ & 0.80 \\
\hline Body mass index $\left(\mathrm{kg} / \mathrm{m}^{2}\right)$ & $24.8 \pm 3.5$ & $24.5 \pm 3.5$ & 0.70 \\
\hline Education (y) & $15.5 \pm 5.7$ & $16.1 \pm 4.4$ & 0.67 \\
\hline \multicolumn{4}{|l|}{ Temperament traits ( $\mathrm{TCl}$ scores) } \\
\hline Novelty seeking & $19.1 \pm 6.7$ & $16.2 \pm 4.8$ & 0.093 \\
\hline Harm avoidance & $20.6 \pm 9.2$ & $13.3 \pm 5.6$ & 0.001 \\
\hline Reward dependence & $15.8 \pm 4.7$ & $17.5 \pm 4.2$ & 0.22 \\
\hline Persistence & $4.7 \pm 2.4$ & $4.9 \pm 2.1$ & 0.85 \\
\hline Family history of alcoholism ( $n$ ) & 13 & 0 & $<0.001$ \\
\hline \multicolumn{4}{|l|}{ Alcohol use } \\
\hline Reported intake (g/wk) & $1,627 \pm 648$ & $24 \pm 21$ & $<0.001$ \\
\hline Hair ethyl glucuronide level $(\mathrm{pg} / \mathrm{mg})$ & $221.5(375.1)$ & $8.1(12.2)$ & $<0.001$ \\
\hline AUDIT score & $27 \pm 4$ & $2 \pm 1$ & $<0.001$ \\
\hline Reported use (y) & $26.5 \pm 12.9$ & $26.6 \pm 8.1$ & 0.99 \\
\hline Reported problematic use (y) & $12.7 \pm 10.7$ & - & - \\
\hline Abstinence (d) & $7.3 \pm 4.0$ & - & - \\
\hline \multicolumn{4}{|l|}{ Alcohol craving (DDQ scores) } \\
\hline Desire and intention & $15.7 \pm 7.0$ & - & - \\
\hline Negative reinforcement & $14.8 \pm 6.5$ & - & - \\
\hline Control & $4.0(5.0)$ & - & - \\
\hline Nicotine use $(n)$ & 11 & 0 & $<0.001$ \\
\hline Number of cigarettes per day & $18.5(20)$ & - & - \\
\hline Regular use $(y)$ & $23 \pm 6$ & - & - \\
\hline FTND score & $5.5 \pm 2.7$ & - & - \\
\hline \multicolumn{4}{|l|}{${ }^{18} \mathrm{~F}-\mathrm{FPEB}$} \\
\hline Injected activity (MBq) & $175 \pm 11$ & $177.0 \pm 7$ & $>0.5$ \\
\hline Specific activity $(\mathrm{MBq} / \mathrm{nmol})$ & $97 \pm 51$ & $90 \pm 52$ & $>0.5$ \\
\hline Injected mass $(\mu \mathrm{g})$ & $0.6 \pm 0.4$ & $0.6 \pm 0.6$ & $>0.5$ \\
\hline
\end{tabular}

$\mathrm{TCI}=$ Temperament and Character Inventory; AUDIT = Alcohol Use Disorders Identification Test; DDQ = Desires for Drug Questionnaire adapted for alcohol; FTND = Fagerstrom Test for Nicotine Dependence. Continuous data are mean \pm SD or median followed by interquartile range in parentheses. Dichotomous data are numbers. 
(29). Moreover, alcohol consumption was quantitatively assessed via hair ethyl glucuronide analysis (30), providing a measure of alcohol consumption over the prior 3 mo (31).

Alcohol craving in recently abstinent subjects was assessed at the time of PET using the 13-item Dutch version of the Desires for Drug Questionnaire adapted to alcohol (32).

\section{mGlu5 PET Imaging and Radiometabolite Analysis}

All subjects were asked to fast for $3 \mathrm{~h}$ before undergoing imaging. Lack of alcohol consumption in the prior hours was verified by measuring the breath alcohol concentration using a hand-held electronic device (Alcotest 6820; Dräger). The radiometabolite analysis procedure has been described in detailed previously (20).

${ }^{18} \mathrm{~F}-\mathrm{FPEB}$ PET data were dynamically acquired on a HiRez Biograph PET/CT camera (Siemens) for $90 \mathrm{~min}$ after a bolus injection of ${ }^{18} \mathrm{~F}-\mathrm{FPEB}$ (Table 1). PET data were analyzed using PMOD software (version 3.605, PMOD Technologies) and statistical parametric mapping (SPM, version 12). The ${ }^{18} \mathrm{~F}$-FPEB total distribution volume $\left(\mathrm{V}_{\mathrm{T}}\right.$, as defined by Innis et al. (33)) was used as a surrogate for mGlu5 availability. To obtain a $V_{T}$ map, the Logan graphical approach (34) was used. For SPM analysis, $\mathrm{V}_{\mathrm{T}}$ images were spatially normalized to the Montreal Neurologic Institute space. Additionally, voxel-based findings were corroborated with a volume-ofinterest (VOIs) analysis using the N30R83 Hammers probabilistic atlas (35). VOI-based $\mathrm{V}_{\mathrm{T}}$ values were derived from a reversible 2tissue-compartment model $(20,36)$. To control for the confounding effect of potential brain atrophy, voxel-based and VOI-based PET data were corrected for partial-volume effects using Müller-Gärtner (37) and Geometric Transfer Matrix (38) methods, respectively.

\section{Structural MRI and Brain \\ Atrophy Assessment}

Structural brain MRI was performed on a 3-T scanner (Ingenia; Philips Healthcare) for automatic VOI determination and coregistration with PET images. MRI data were acquired with an 8-channel receiver head coil using a volumetric high-resolution T1-weighted magnetization-prepared rapid gradient-echo sequence (repetition time, $9.6 \mathrm{~ms}$; echo time, $4.6 \mathrm{~ms}$; flip angle, $8^{\circ}$; voxel size, $0.98 \times 0.98 \times$ $1.2 \mathrm{~mm})$.

Additionally, potential brain atrophy in subjects was assessed both at the voxel level (voxelbased morphometry, VBM) and at the VOI level by comparing groups' relative gray matter volumes. T1-weighted MR images were first checked for orientation and alignment. VBM was performed with SPM, version 12, using the VBM8 Toolbox. Different tissue types (i.e., gray matter, white matter, and cerebrospinal fluid) were automatically determined in the segmentation process. The gray matter and white matter from all scans were simultaneously iteratively aligned to a study-specific template using "diffeomorphic anatomical registration through exponential lie" algebra. The resulting aligned tissues were spatially normalized to Montreal
Neurological Institute space using high-dimensional warping, then were Jacobian-scaled to account for volume change during the normalization process and to preserve absolute tissue volumes, and finally were spatially smoothed with a Gaussian kernel with a full width at half maximum of $10 \mathrm{~mm}$. These processed gray matter images were then entered into a general linear model, and a 2-sample $t$ test was performed for whole-brain group comparison. Total intracranial volume was used in the statistical design to consider how the regional volumes are likely to vary as a function of whole-brain volume. Total intracranial volume was calculated by adding the volumes of gray matter, white matter, and cerebrospinal fluid using a validated method. Moreover, to corroborate VBM findings, volumetric group differences were also evaluated at a VOI-based level. Gray matter volumes from the Hammers atlas VOIs (35) were extracted after segmentation of the T1-weighed MR images within native MRI space in the PMOD Neuro Tool. Like the voxelwise assessment of volumetric differences, in each subject VOI-based gray matter volumes were normalized to the total intracranial volume.

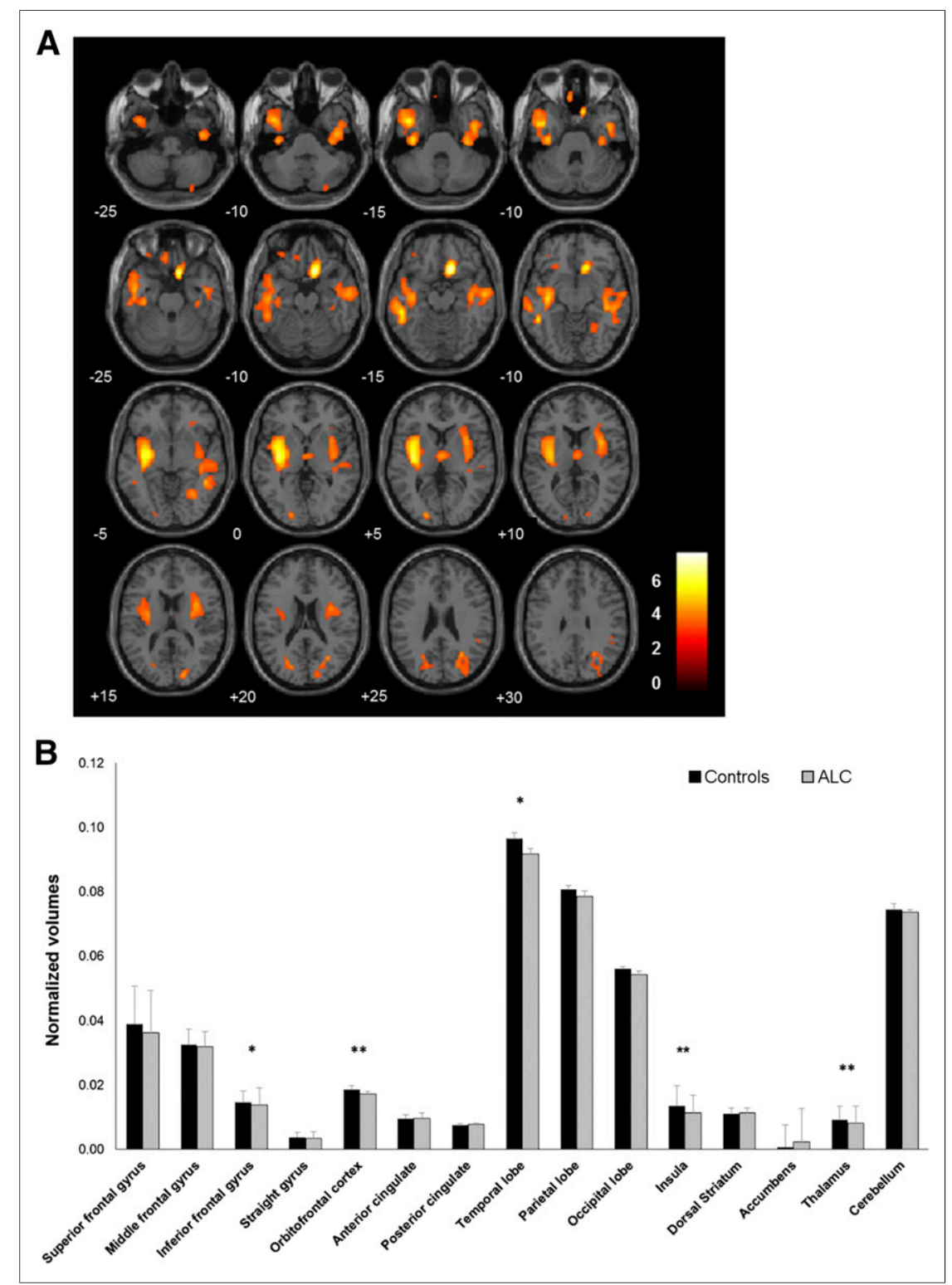

FIGURE 1. Lower gray matter volumes in alcohol-dependent subjects than in controls. (A) SPM displayed at $P<0.001$ (cluster size extent [number of $2 \times 2 \times 2 \mathrm{~mm}$ voxels] $>50$ voxels). (B) VOI analysis. $\mathrm{ACL}=$ alcohol-dependent subject. ${ }^{\star} P<0.05$. ${ }^{*} P<0.001$. 


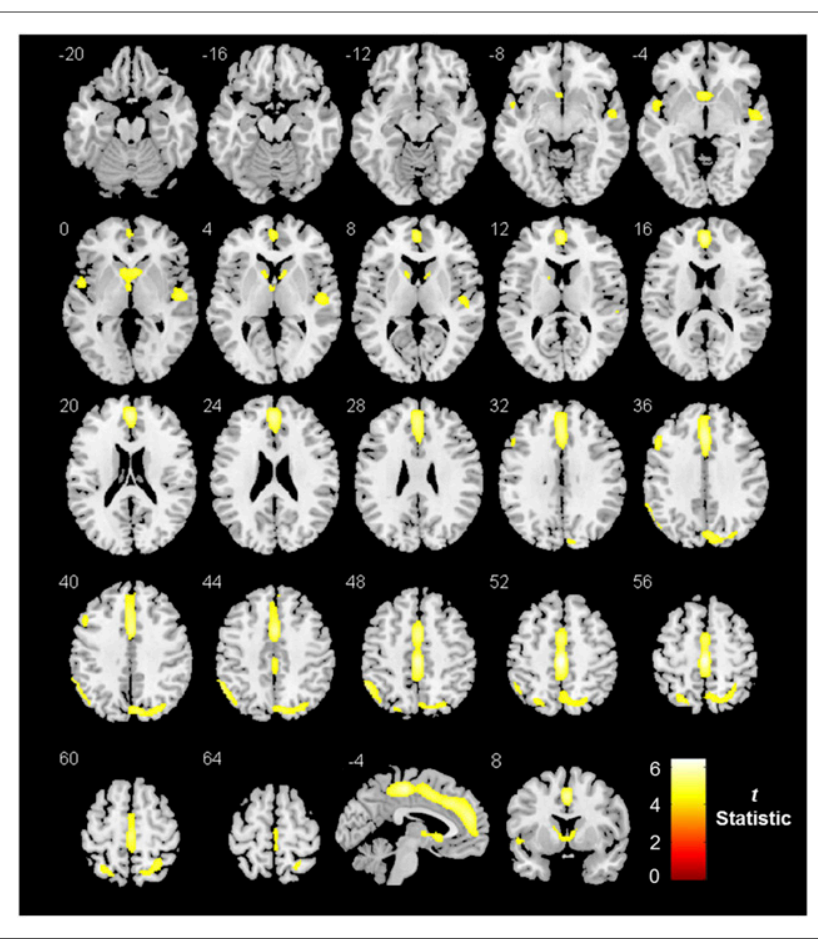

FIGURE 2. Lower mGlu5 availability $\left({ }^{18} \mathrm{~F}-\mathrm{FPEB} \mathrm{V}_{\mathrm{T}}\right)$ in alcohol-dependent subjects than in controls. SPM is displayed at $P<0.05$, corrected for familywise error (cluster size extent [number of $2 \times 2 \times 2 \mathrm{~mm}$ voxels] $>200$ voxels).

\section{Genotyping}

On the basis of previous studies $(23,24)$, the genotypes for mGlu5 polymorphisms rs3462, rs3824927, rs308787, rs11020526, rs7931721, and rs495695 were determined for all participants. Genomic DNA was isolated from $4 \mathrm{~mL}$ of human whole blood using the Chemagic Magnetic Separation Module I (Perkin Elmer) and the according Chemagic
DNA Blood 4k Kit. DNA concentration and purity were measured using a DropSense96 (Trinean) with cDrop software. From each sample, $40 \mu \mathrm{L}$ of DNA $(75 \mathrm{ng} / \mu \mathrm{l})$ were transferred into 96 -well plates. The following primer pairs targeting mGluR5 polymorphisms were developed: 5' CTATGCTCTTCACCCTCCGT $3^{\prime}$ and 5' TTCACACACACACAATGGCA $3^{\prime}$ for rs 3462 , 5' ACAAACTCAAAATCTGGGACCA $3^{\prime}$ and 5' CGCAGCACTCACTATCCAG 3' for rs3824927, 5' CAGGTGGGCTGAGATCTCTT 3' and 5' ACACATCACTCGGAATTGCT $3^{\prime}$ for rs11020526, 5' CATGGGATGAAGACAGAGCTAT $3^{\prime}$ and 5' TCCTAAGCTTCACTCACTGACT $3^{\prime}$ for rs495695, and 5' AGCCAGTTCTAACCTAGGTGT 3' and 5' CATAACAAGCGAGTCTGCCC 3' for rs7931721. The primers were ordered with specific adaptor sequences at the $5^{\prime}$ end, and a first target-specific polymerase chain reaction was performed using the Qiagen Multiplex Kit. Primer-dimers were removed using solid-phase reversible immobilization bead technology with a DNA size cutoff of $200 \mathrm{bp}$. A second polymerase chain reaction was performed with dual indexing primers annealing at the adaptor sequence of products of the first polymerase chain reaction. Using solid-phase reversible immobilization bead technology with a DNA size cutoff of $250 \mathrm{bp}$, primer-dimers were removed. The samples were pooled at a concentration of $2 \mathrm{nM}$. Sequencing was performed using v3 paired-end 300-bp sequencing on the Illumina MiSeq platform.

\section{Statistical Analysis}

Conventional statistical analyses were performed using Statistica, version 12 (Statsoft). Normally distributed variables are reported as mean $\pm \mathrm{SD}$, and skewed variables, as median and interquartile range. Independent-sample $t$ tests or Mann-Whitney $U$ tests were used, as appropriate. Because of the suggested involvement of mGlu5 in normal aging $(21,39)$, age was included as a covariate in all analyses.

A SPM group-comparison analysis was performed with smoking status as a nuisance variable. $\mathrm{V}_{\mathrm{T}}$ images corrected for partial-volume effects were smoothed using a gaussian kernel of $12 \mathrm{~mm}$. The statistical threshold was set at a $P$ value of less than 0.05 , corrected for familywise error. To minimize the chance of type I error in the secondary VOI-based analysis, only VOIs corresponding to regions with a

TABLE 2

SPM of Lower mGlu5 Availability in Alcohol-Dependent Subjects vs. Controls

\begin{tabular}{|c|c|c|c|c|c|c|c|c|c|}
\hline \multicolumn{2}{|c|}{ Cluster level } & \multicolumn{2}{|c|}{ Voxel level } & \multicolumn{3}{|c|}{$\begin{array}{l}\text { Peak voxel MNI } \\
\text { coordinate }\end{array}$} & \multicolumn{3}{|l|}{ Location } \\
\hline $\mathrm{K}_{\mathrm{EXT}}$ & $P^{\star}$ & $t$ score & $P^{*}$ & $x$ & $y$ & $z$ & Anatomic area & Hemisphere & BA \\
\hline \multirow[t]{3}{*}{7,210} & $<0.001$ & 6.45 & $<0.001$ & 2 & -26 & 53 & Precentral gyrus & Right & 4 \\
\hline & & 5.82 & 0.001 & 2 & 48 & 21 & Anterior cingulate gyrus & Right & 32 \\
\hline & & 5.78 & 0.001 & 2 & 27 & 36 & Anterior cingulate gyrus & Right & 24 \\
\hline 711 & 0.007 & 5.46 & 0.003 & 54 & -54 & 48 & Parietal lobe, inferolateral remainder & Right & 40 \\
\hline \multirow[t]{2}{*}{748} & 0.001 & 5.06 & 0.008 & -6 & -78 & 39 & Superior parietal gyrus & Left & 39 \\
\hline & & 4.80 & 0.017 & -29 & -75 & 42 & Occipital lobe, lateral remainder & Left & 7 \\
\hline \multirow[t]{3}{*}{874} & 0.006 & 4.97 & 0.011 & -6 & 12 & 2 & Caudate nucleus & Left & - \\
\hline & & 4.88 & 0.014 & 5 & 14 & -3 & Caudate nucleus & Right & - \\
\hline & & 4.72 & 0.022 & 3 & -3 & 2 & Thalamus & Right & - \\
\hline 266 & 0.019 & 4.90 & 0.013 & 50 & 20 & 36 & Middle frontal gyrus & Right & 44 \\
\hline 770 & 0.007 & 4.88 & 0.014 & -51 & -14 & 2 & Superior temporal gyrus, posterior & Left & 48 \\
\hline 355 & 0.015 & 4.86 & 0.015 & 24 & -66 & 57 & Superior parietal gyrus & Right & 7 \\
\hline 290 & 0.018 & 4.72 & 0.021 & 53 & 2 & -2 & Superior temporal gyrus, posterior & Right & 38 \\
\hline
\end{tabular}

${ }^{*}$ Corrected for familywise error.

$\mathrm{MNI}=$ Montreal Neurological Institute; $\mathrm{K}_{\mathrm{EXT}}=$ cluster size extent (number of $2 \times 2 \times 2 \mathrm{~mm}$ voxels); $\mathrm{BA}=$ Brodmann area. 


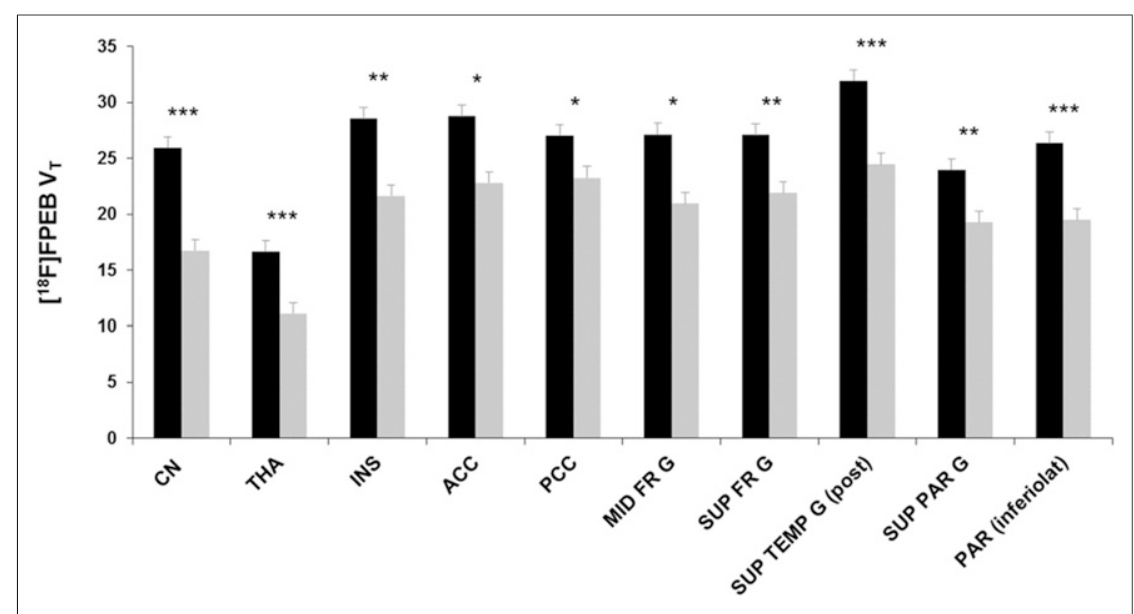

FIGURE 3. VOI analysis of mGlu5 availability $\left({ }^{18} \mathrm{~F}-\mathrm{FPEB} \mathrm{V}_{\mathrm{T}}\right)$ in controls (black bars) vs. alcoholdependent subjects (gray bars). Bars and error bars are mean and SD, respectively. $\mathrm{CN}=$ caudate nucleus; THA = thalamus; INS = insula; $A C C=$ anterior cingulate cortex; $P C C=$ posterior cingulate cortex; MID FR G = middle frontal gyrus; SUP FR G = superior frontal gyrus; SUP TEMP G (post) = superior temporal gyrus, posterior part; SUP PAR G = superior parietal gyrus; PAR (inferolat) = parietal lobe, inferolateral part. ${ }^{\star} P<0.05 .{ }^{\star \star} P<0.01$. ${ }^{\star \star \star} P<0.001$. measurements were assessed using partial correlation coefficients with age as a nuisance variable since both mGlu5 density (21) and alcohol craving (40) may be influenced by age.

\section{RESULTS}

\section{Participant Characteristics}

The participant characteristics are reported in Table 1. The two groups differed only in alcohol and nicotine use and in the temperament trait "harm avoidance."

\section{Brain Atrophy in Subjects}

Compared with controls, VBM revealed bilaterally lower gray matter volume in alcohol-dependent subjects in the orbitofrontal cortex; straight gyrus; inferior, middle, and superior temporal lobes; hippocampus; insula; and putamen (Fig. 1A). Consistently, evidence of brain atrophy in alcohol-dependent subjects was observed in the additional VOIbased analysis (Fig. 1B). significant familywise error-corrected $P$ value were considered. VOI-based group comparisons were analyzed using a multivariate general linear model with ${ }^{18} \mathrm{~F}$-FPEB $\mathrm{V}_{\mathrm{T}}$ values as dependent variables and group status, smoking status, and age as independent predictors. The effect of mGlu5 polymorphisms, withdrawal treatment, and abstinence period were tested by adding genotyping data, the received benzodiazepine dose, and days of abstinence, respectively, into the model.

In subjects, associations between VOI-based mGlu5 availability and self-reported alcohol use, hair ethyl glucuronide levels, and craving

\section{Group Differences in mGlu5 Availability}

All scans were obtained at least $2 \mathrm{~d}$ from the last alcohol consumption (mean interval, $7 \mathrm{~d}$; range, 2-14 d) and more than $24 \mathrm{~h}$ from the last clorazepate dosing (mean interval, $91 \mathrm{~h}$; range, 25-186 h).

After correction for partial-volume effects and controlling for age and smoking status, SPM showed a significantly lower mGlu5 availability in alcohol-dependent subjects in variousmostly limbic, cortical, and subcortical-brain areas (Fig. 2; Table 2). These findings were confirmed by the VOI analysis, detecting a lower mGlu5 availability in alcohol-dependent

TABLE 3

Partial Correlation Coefficients* of mGluR Availability $\left({ }^{18} \mathrm{~F}-\mathrm{FPEB} \mathrm{V}_{\mathrm{T}}\right)$ vs. Recent Alcohol Consumption and Craving in Alcohol-Dependent Subjects

\begin{tabular}{|c|c|c|c|c|c|c|c|c|}
\hline \multirow[b]{3}{*}{ Volume of interest } & & & \multicolumn{6}{|c|}{ Alcohol craving (DDQ) } \\
\hline & \multicolumn{2}{|c|}{$\begin{array}{l}\text { hEtG level } \\
(\mathrm{pg} / \mathrm{mg})\end{array}$} & \multicolumn{2}{|c|}{$\begin{array}{l}\text { Desire and } \\
\text { intention }\end{array}$} & \multicolumn{2}{|c|}{$\begin{array}{c}\text { Negative } \\
\text { reinforcement }\end{array}$} & \multicolumn{2}{|c|}{ Control } \\
\hline & $r$ & $P$ & $r$ & $P$ & $r$ & $P$ & $r$ & $P$ \\
\hline Caudate nucleus & -0.38 & 0.20 & 0.05 & 0.86 & 0.10 & 0.73 & -0.15 & 0.58 \\
\hline Thalamus & -0.65 & 0.03 & 0.00 & 1.00 & 0.48 & 0.07 & 0.16 & 0.57 \\
\hline Insula & -0.58 & 0.04 & -0.21 & 0.46 & 0.49 & 0.06 & 0.24 & 0.40 \\
\hline Anterior cingulate cortex & -0.43 & 0.04 & -0.14 & 0.61 & 0.52 & 0.05 & 0.26 & 0.36 \\
\hline Posterior cingulate cortex & -0.45 & 0.12 & 0.04 & 0.88 & 0.57 & 0.03 & 0.18 & 0.51 \\
\hline Middle frontal gyrus & -0.50 & 0.08 & -0.10 & 0.73 & 0.59 & 0.02 & 0.18 & 0.52 \\
\hline Superior frontal gyrus & -0.57 & 0.04 & -0.08 & 0.78 & 0.50 & 0.06 & 0.08 & 0.77 \\
\hline Superior temporal gyrus (posterior) & -0.58 & 0.04 & -0.18 & 0.52 & 0.43 & 0.11 & 0.02 & 0.95 \\
\hline Superior parietal gyrus & -0.56 & 0.04 & -0.03 & 0.91 & 0.50 & 0.06 & 0.09 & 0.76 \\
\hline Parietal lobe (inferolateral) & -0.52 & 0.06 & -0.02 & 0.95 & 0.53 & 0.04 & 0.05 & 0.85 \\
\hline Mean $(n=10$ VOls $)$ & -0.56 & 0.04 & -0.09 & 0.76 & 0.53 & 0.04 & 0.11 & 0.69 \\
\hline
\end{tabular}

*With age as nuisance variable.

$\mathrm{hEtG}=$ hair ethyl glucuronide; DDQ = Desires for Drug Questionnaire adapted for alcohol. 
subjects ranging from $14 \%$ (posterior cingulate cortex) to $36 \%$ (caudate nucleus) (Fig. 3). Noteworthy, no significant ${ }^{18} \mathrm{~F}$ FPEB $V_{T}$ differences were observed between smokers and nonsmokers in alcohol-dependent subjects in any region (global $\mathrm{V}_{\mathrm{T}}, 22 \pm 4$ in smokers and $19 \pm 2$ in nonsmokers; $P=0.8$ ). Finally, similar highly significant ${ }^{18} \mathrm{~F}-\mathrm{FPEB} \quad \mathrm{V}_{\mathrm{T}}$ differences were observed using data uncorrected for partial-volume effects, supporting that group differences in brain volume did not affect the present PET findings. No significant sex differences in mGlu5 availability were observed, conforming with previous studies $(21,41)$. Regarding mGlu5 polymorphisms, we found no significant effects of an individual polymorphism genotype on ${ }^{18} \mathrm{~F}$-FPEB $\mathrm{V}_{\mathrm{T}}$ in either the control group or the alcoholdependent group.

\section{Correlation Between mGlu5 Availability and Alcohol Consumption}

In alcohol-dependent subjects, mGlu5 availability in the thalamus, insula, superior frontal gyrus, superior temporal gyrus, and inferolateral temporal lobe correlated negatively with hair ethyl glucuronide concentration (Table 3; Fig. 4A). Concordant findings were observed at the voxel level using multiple regression analysis, except for the thalamus $(P<0.001$; Fig. 5). In contrast, no associations were found between mGlu5 and the self-reported

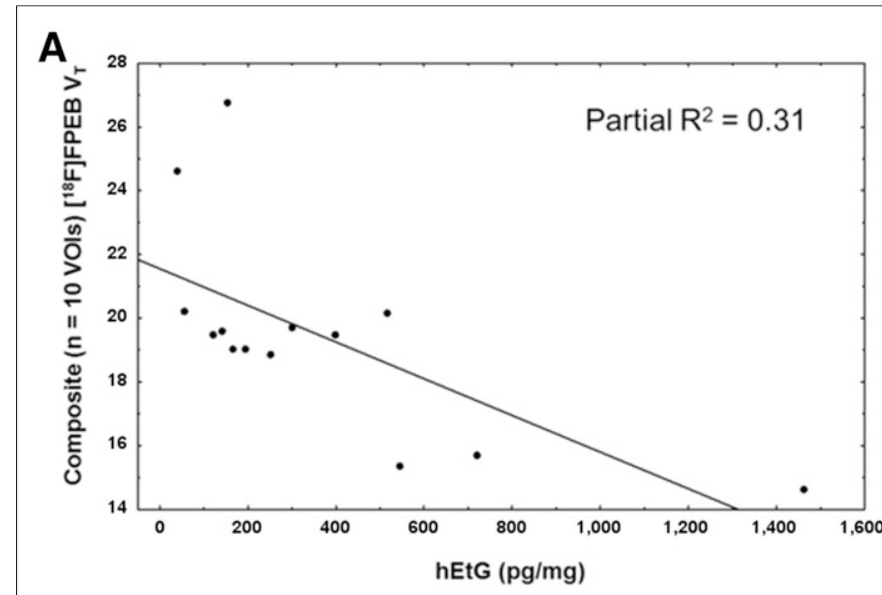

B

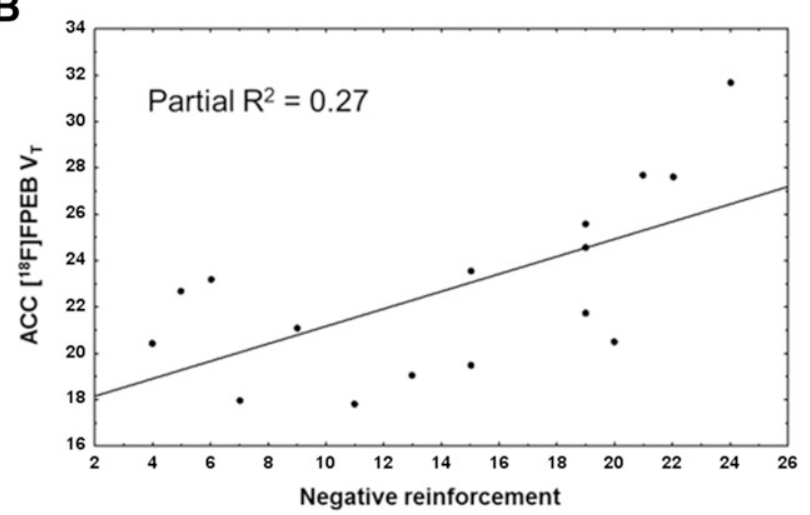

D

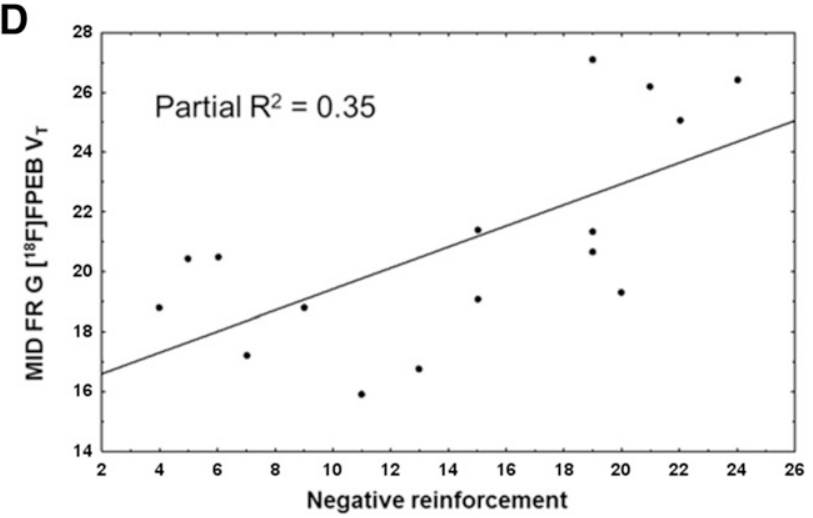

C

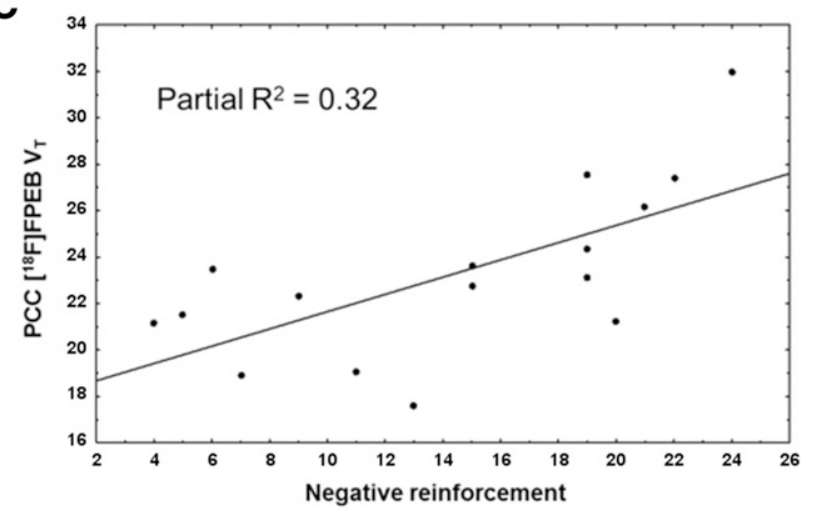

E

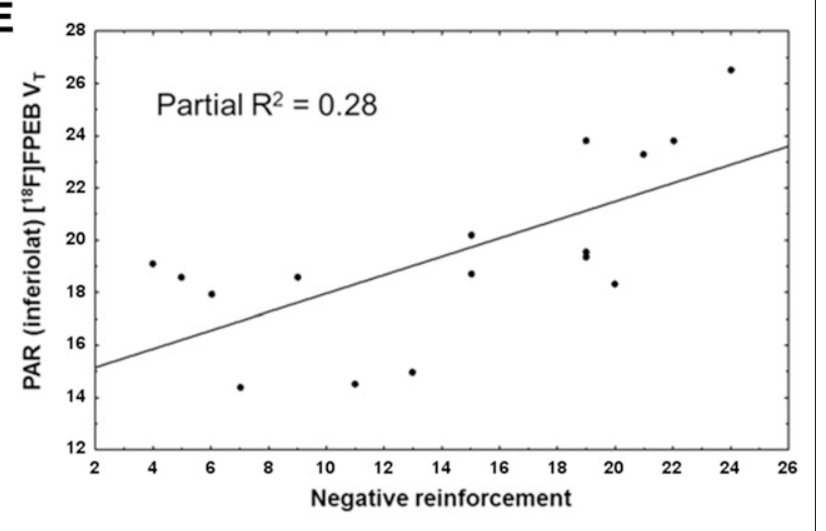

FIGURE 4. Alcohol-dependent subjects' scatterplots showing relation between mGlu5 availability $\left({ }^{18} \mathrm{~F}-\mathrm{FPEB} \mathrm{V}_{\mathrm{T}}\right.$ ) and hair ethyl glucuronide ( $\mathrm{hEtG}$ ) levels at mean composite VOI (A) and between mGlu5 availability and negative reinforcement craving score for anterior cingulate (B), posterior cingulate $(C)$, middle frontal gyrus $(D)$, and inferolateral temporal lobe $(E)$. 


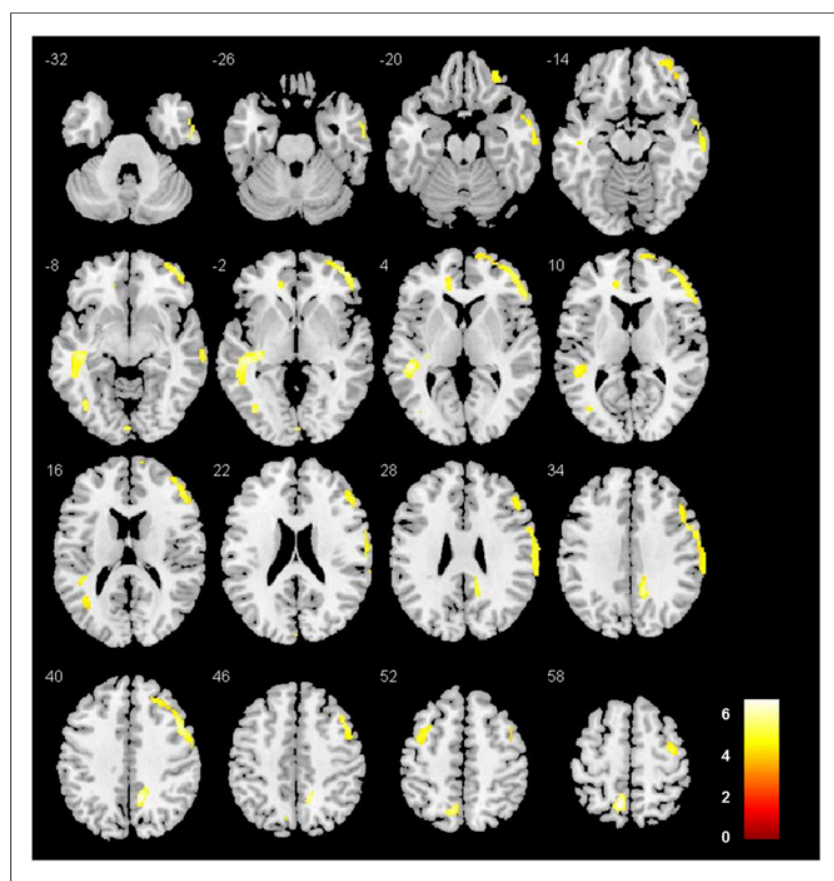

FIGURE 5. SPM showing negative association between mGlu5 availability $\left({ }^{18} \mathrm{~F}\right.$-FPEB $\left.\mathrm{V}_{\mathrm{T}}\right)$ and hair ethyl glucuronide levels in alcoholdependent subjects $(P<0.001$, cluster size extent [number of $2 \times 2 \times$ $2 \mathrm{~mm}$ voxels] $>50$ voxels).

alcohol consumption assessments. Additionally, no associations between mGlu5 availability and the duration of alcohol consumption or the time of abstinence were observed.

\section{Correlation Between mGlu5 Availability and Alcohol Craving}

Using age as a nuisance variable, VOI-based mGlu5 availability in alcohol-dependent subjects correlated positively with the craving item "negative reinforcement" in the cingulate cortex, middle frontal gyrus, and inferolateral parietal lobe (Table 3; Figs. 4B-4E). These results did not survive a Bonferroni adjustment for multiple testing and were not reproduced by SPM analysis, possibly because of the low effect size and the reduced sensitivity of the SPM compared with the VOI-based analysis, respectively.

\section{DISCUSSION}

To our knowledge, this was the first study investigating mGlu5 availability in alcohol-dependent subjects in vivo. Within $2 \mathrm{wk}$ of supervised abstinence, alcohol-dependent subjects demonstrated lower mGlu5 availability in mainly limbic areas. Moreover, mGlu5 availability was inversely correlated with the quantitative measure of recent alcohol consumption. Finally, we found a positive relationship between mGlu5 and alcohol craving during early abstinence, supporting the clinical significance of the present findings.

In alcohol-dependent subjects, we observed reduced mGlu5 availability in corticolimbic regions, in which long-lasting neuroadaptations are suggested to occur on extensive alcohol exposure $(1,26)$. A lower cerebral mGlu5 availability was also observed in nicotine- (42) and cocaine-dependent (43) subjects, supporting the hypothesis that mGlu5 downregulation represents a common neuroadaptation occurring across various types of substance dependence. In alcohol dependence, a well-described neuroadaptation is the upregulation of $N$-methyl-D-aspartate receptor expression and function, which contribute to the hyperglutamatergic state associated with short-term abstinence (44) and, in turn, to neurotoxicity (45), anxiety (46), craving (47), and relapse (48). Therefore, given the functional positive coupling of mGlu5 and $\mathrm{N}$-methyl-D-aspartate receptor (3), mGlu5 availability might serve as a compensatory mechanism to attenuate this hyperglutamatergic state by reducing postsynaptic excitability. On the other hand, given its critical role in adaptive learning (49), impaired mGlu5-dependent plasticity may contribute to the chronic inability of dependent individuals to extinguish drug-associated memories and to form adaptive behaviors to escape drug seeking and taking $(50,51)$.

In alcohol-dependent subjects, the finding of a negative association between hair ethyl glucuronide concentrations and mGlu5 suggests a dose-response relationship to alcohol exposure. In contrast, no significant associations were found with self-reported alcohol measures. This discrepancy might be due to the difficulty in recalling accurate amounts of consumed alcohol in the presence of cognitive impairment or to the well-known tendency to underestimate or inaccurately report one's own alcohol use (52).

An important consideration to address is whether the present findings have some clinical implications. A fundamental component of alcohol dependence is craving. Using a subjective assessment of craving (53), we found a positive relationship between the craving dimension "negative reinforcement" and mGlu5 availability in specific regions that are involved in controlling drug craving $(54,55)$. This association between mGlu5 and craving is also in line with preclinical evidence showing that antagonism of mGlu5 reduces the escalation of alcohol use as well as anxietylike (56) and relapselike (57) behaviors. Therefore, our findings support the potential of specific mGlu5 negative modulators to reduce craving and relapse in alcohol-dependent subjects (58).

Some limitations of this study need to be mentioned. First, the sample size of alcohol-dependent subjects was small, though large enough to draw robust conclusions regarding group difference in mGlu5 availability. In contrast, the correlations with craving showed a small effect size, and this finding should be seen as preliminary. Second, considering the hyperglutamatergic state associated with alcohol withdrawal (26), group differences in extracellular glutamate levels potentially confound the findings. Indeed, although binding to an allosteric site, radioligand displacement by endogenous glutamate might occur by mechanisms other than direct competition (59). Yet, we previously found no association between anterior cingulate ${ }^{18} \mathrm{~F}-\mathrm{FPEB} \mathrm{V}_{\mathrm{T}}$ and glutamate levels, supporting no major effects of endogenous glutamate on ${ }^{18} \mathrm{~F}-\mathrm{FPEB}$ binding. Third, this study cannot disentangle whether the reduced mGlu5 availability in alcohol-dependent subjects represents a preexistent condition or a consequence of chronic alcohol use, which would be better investigated in longitudinal studies. Finally, mGlu5 scans have been performed within a rather large withdrawal time window (2-14 d). In view of the preclinical literature suggesting that mGlu5 expression varies depending on the time of abstinence and the brain regions being studied (6-9), more studies are needed to investigate how mGlu5 expression regionally adapts over time in alcohol-dependent subjects.

\section{CONCLUSION}

We found that alcohol-dependent subjects have lower corticolimbic mGlu5 availability than controls. In specific regions, this reduction correlated negatively with the amount of recent alcohol consumed and positively with the negative reinforcement dimension of craving. These 
findings provide in vivo human evidence for a central role of mGlu5 in the neuroadaptations underlying alcohol dependence and support the potential of mGlu5 antagonists for the prevention or reduction of craving in alcohol-dependent subjects. Future follow-up mGlu5 PET studies in the course of alcohol detoxification are warranted to assess both the potential reversibility of lower mGlu5 availability and the potential of mGlu5 PET for individual prediction of relapse.

\section{DISCLOSURE}

Koen Van Laere is a senior clinical research fellow for the Research Foundation-Flanders (FWO) and has received an FWO research grant for this work (FWO/G.0548.06). Jenny Ceccarini is a FWO postdoctoral fellow. Bart de Laat received a scholarship from the Flemish Agency for Innovation (IWT). No other potential conflict of interest relevant to this article was reported.

\section{ACKNOWLEDGMENTS}

We acknowledge Kwinten Porters, Jef Van Loock, the radiopharmacy and radiology team at UZ Leuven, the Toxicological Center of Antwerp University, and the Toxicology and TDM Laboratory of ZNA Stuivenberg Hospital.

\section{REFERENCES}

1. Kalivas PW. The glutamate homeostasis hypothesis of addiction. Nat Rev Neurosci. 2009;10:561-572.

2. Patel S, Hamill TG, Connolly B, Jagoda E, Li W, Gibson RE. Species differences in mGluR5 binding sites in mammalian central nervous system determined using in vitro binding with $\left[{ }^{18}\right.$ F]F-PEB. Nucl Med Biol. 2007;34:1009-1017.

3. Sengmany K, Gregory KJ. Metabotropic glutamate receptor subtype 5: molecular pharmacology, allosteric modulation and stimulus bias. $\mathrm{Br} J$ Pharmacol. 2016;173:3001-3017.

4. Carroll FI. Antagonists at metabotropic glutamate receptor subtype 5: structure activity relationships and therapeutic potential for addiction. Ann N Y Acad Sci. 2008;1141:221-232.

5. Rehm J, Mathers C, Popova S, Thavorncharoensap M, Teerawattananon Y, Patra J. Global burden of disease and injury and economic cost attributable to alcohol use and alcohol-use disorders. Lancet. 2009;373:2223-2233.

6. Simonyi A, Christian MR, Sun AY, Sun GY. Chronic ethanol-induced subtypeand subregion-specific decrease in the mRNA expression of metabotropic glutamate receptors in rat hippocampus. Alcohol Clin Exp Res. 2004;28:1419-1423.

7. Kumar J, Hapidin H, Get Bee YT, Ismail Z. The effects of acute ethanol administration on ethanol withdrawal-induced anxiety-like syndrome in rats: a biochemical study. Alcohol. 2016;50:9-17.

8. Szumlinski KK, Ary AW, Lominac KD, Klugmann M, Kippin TE. Accumbens Homer2 overexpression facilitates alcohol-induced neuroplasticity in C57BL/6J mice. Neuropsychopharmacology. 2008;33:1365-1378.

9. Obara I, Bell RL, Goulding SP, et al. Differential effects of chronic ethanol consumption and withdrawal on Homer/glutamate receptor expression in subregions of the accumbens and amygdala of P rats. Alcohol Clin Exp Res. 2009;33: 1924-1934.

10. Schroeder JP, Overstreet DH, Hodge CW. The mGluR5 antagonist MPEP decreases operant ethanol self-administration during maintenance and after repeated alcohol deprivations in alcohol-preferring (P) rats. Psychopharmacology (Berlin). 2005;179:262-270.

11. Gupta T, Syed YM, Revis AA, et al. Acute effects of acamprosate and MPEP on ethanol drinking-in-the-dark in male C57BL/6J mice. Alcohol Clin Exp Res. 2008;32:1992-1998.

12. Besheer J, Faccidomo S, Grondin JJ, Hodge CW. Regulation of motivation to self-administer ethanol by mGluR5 in alcohol-preferring (P) rats. Alcohol Clin Exp Res. 2008;32:209-221.

13. Kotlinska JH, Bochenski M, Danysz W. The role of group I mGlu receptors in the expression of ethanol-induced conditioned place preference and ethanol withdrawal seizures in rats. Eur J Pharmacol. 2011;670:154-161.
14. Besheer J, Grondin JJ, Salling MC, Spanos M, Stevenson RA, Hodge CW. Interoceptive effects of alcohol require mGlu5 receptor activity in the nucleus accumbens. J Neurosci. 2009;29:9582-9591.

15. Blednov YA, Harris RA. Metabotropic glutamate receptor 5 (mGluR5) regulation of ethanol sedation, dependence and consumption: relationship to acamprosate actions. Int J Neuropsychopharmacol. 2008;11:775-793.

16. Lee JY, Choe ES, Yang CH, et al. The mGluR5 antagonist MPEP suppresses the expression and reinstatement, but not the acquisition, of the ethanol-conditioned place preference in mice. Pharmacol Biochem Behav. 2016;140:33-38.

17. Schroeder JP, Spanos M, Stevenson JR, Besheer J, Salling M, Hodge CW. Cueinduced reinstatement of alcohol-seeking behavior is associated with increased ERK1/2 phosphorylation in specific limbic brain regions: blockade by the mGluR5 antagonist MPEP. Neuropharmacology. 2008;55:546-554.

18. Adams CL, Short JL, Lawrence AJ. Cue-conditioned alcohol seeking in rats following abstinence: involvement of metabotropic glutamate 5 receptors. $\mathrm{Br} \mathrm{J}$ Pharmacol. 2010;159:534-542.

19. Reynolds AR, Williams LA, Saunders MA, Prendergast MA. Group 1 mGlufamily proteins promote neuroadaptation to ethanol and withdrawal-associated hippocampal damage. Drug Alcohol Depend. 2015;156:213-220.

20. Leurquin-Sterk G, Postnov A, de Laat B, et al. Kinetic modeling and long-term test-retest reproducibility of the mGluR5 PET tracer ${ }^{18} \mathrm{~F}-\mathrm{FPEB}$ in human brain. Synapse. 2016;70:153-162.

21. Leurquin-Sterk G, Van den Stock J, Crunelle CL, et al. Positive association between limbic metabotropic glutamate receptor 5 availability and noveltyseeking temperament in humans: a ${ }^{18}$ F-FPEB PET study. J Nucl Med. 2016;57:17461752 .

22. Cloninger CR. Neurogenetic adaptive mechanisms in alcoholism. Science. 1987; 236:410-416.

23. Meyers JL, Salling MC, Almli LM, et al. Frequency of alcohol consumption in humans: the role of metabotropic glutamate receptors and downstream signaling pathways. Transl Psychiatry. 2015;5:e586.

24. Schumann G, Johann M, Frank J, et al. Systematic analysis of glutamatergic neurotransmission genes in alcohol dependence and adolescent risky drinking behavior. Arch Gen Psychiatry. 2008;65:826-838.

25. Fattore L, Diana M. Drug addiction: an affective-cognitive disorder in need of a cure. Neurosci Biobehav Rev. 2016;65:341-361.

26. Gass JT, Olive MF. Glutamatergic substrates of drug addiction and alcoholism. Biochem Pharmacol. 2008;75:218-265.

27. Cloninger CR, Svrakic DM, Przybeck TR. A psychobiological model of temperament and character. Arch Gen Psychiatry. 1993;50:975-990.

28. Sobell LC, Sobell MB. Timeline follow-back: a technique for assessing selfreported ethanol consumption. In: Litten RZ, Allen JP, eds. Measuring Alcohol Consumption: Psychosocial and Biochemical Methods. Totowa, NJ: Humana Press; 1992:41-72.

29. Saunders JB, Aasland OG, Babor TF, de la Fuente JR, Grant M. Development of the Alcohol Use Disorders Identification Test (AUDIT): WHO collaborative project on early detection of persons with harmful alcohol consumption-II. Addiction. 1993;88:791-804.

30. Crunelle CL, Yegles M, van Nuijs AL, et al. Hair ethyl glucuronide levels as a marker for alcohol use and abuse: a review of the current state of the art. Drug Alcohol Depend. 2014;134:1-11.

31. Crunelle CL, Cappelle D, Covaci A, et al. Hair ethyl glucuronide as a biomarker of alcohol consumption in alcohol-dependent patients: role of gender differences. Drug Alcohol Depend. 2014;141:163-166.

32. Franken IH, Hendriksa VM, van den Brink W. Initial validation of two opiate craving questionnaires: the Obsessive Compulsive Drug Use Scale and the Desires for Drug Questionnaire. Addict Behav. 2002;27:675-685.

33. Innis RB, Cunningham VJ, Delforge J, et al. Consensus nomenclature for in vivo imaging of reversibly binding radioligands. J Cereb Blood Flow Metab. 2007;27: 1533-1539.

34. Logan J, Fowler JS, Volkow ND, et al. Graphical analysis of reversible radioligand binding from time-activity measurements applied to $\left[\mathrm{N}-{ }^{11} \mathrm{C}-\right.$ methyl]-(-)cocaine PET studies in human subjects. J Cereb Blood Flow Metab. 1990;10: 740-747.

35. Hammers A, Allom R, Koepp MJ, et al. Three-dimensional maximum probability atlas of the human brain, with particular reference to the temporal lobe. Hum Brain Mapp. 2003;19:224-247.

36. Wong DF, Waterhouse R, Kuwabara H, et al. ${ }^{18}$ F-FPEB, a PET radiopharmaceutical for quantifying metabotropic glutamate 5 receptors: a first-in-human study of radiochemical safety, biokinetics, and radiation dosimetry. J Nucl Med. 2013;54: 388-396.

37. Müller-Gärtner HW, Links JM, Prince JL, et al. Measurement of radiotracer concentration in brain gray matter using positron emission tomography: MRI-based correction for partial volume effects. J Cereb Blood Flow Metab. 1992;12:571-583. 
38. Rousset OG, Ma Y, Evans AC. Correction for partial volume effects in PET: principle and validation. J Nucl Med. 1998;39:904-911.

39. Ménard C, Quirion R. Successful cognitive aging in rats: a role for mGluR5 glutamate receptors, Homer 1 proteins and downstream signaling pathways. PLoS One. 2012;7:e28666.

40. Hintzen AK, Cramer J, Karagulle D, et al. Does alcohol craving decrease with increasing age? Results from a cross-sectional study. J Stud Alcohol Drugs. 2011;72:158-162.

41. DuBois JM, Rousset OG, Rowley J, et al. Characterization of age/sex and the regional distribution of mGluR5 availability in the healthy human brain measured by high-resolution $\left[{ }^{11} \mathrm{C}\right] \mathrm{ABP} 688$ PET. Eur J Nucl Med Mol Imaging. 2016; 43:152-162.

42. Akkus F, Ametamey SM, Treyer V, et al. Marked global reduction in mGluR5 receptor binding in smokers and ex-smokers determined by $\left[{ }^{11} \mathrm{C}\right]$ ABP688 positron emission tomography. Proc Natl Acad Sci USA. 2013;110: 737-742.

43. Martinez D, Slifstein M, Nabulsi N, et al. Imaging glutamate homeostasis in cocaine addiction with the metabotropic glutamate receptor 5 positron emission tomography radiotracer $\left[{ }^{11} \mathrm{C}\right] \mathrm{ABP} 688$ and magnetic resonance spectroscopy. Biol Psychiatry. 2014;75:165-171.

44. Chandrasekar R. Alcohol and NMDA receptor: current research and future direction. Front Mol Neurosci. 2013;6:14.

45. Oka M, Hirouchi M, Tamura M, Sugahara S, Oyama T. Acamprosate \{monocalcium bis(3-acetamidopropane-1-sulfonate)\} reduces ethanol-drinking behavior in rats and glutamate-induced toxicity in ethanol-exposed primary rat cortical neuronal cultures. Eur J Pharmacol. 2013;718:323-331.

46. Barkus C, McHugh SB, Sprengel R, Seeburg PH, Rawlins JN, Bannerman DM. Hippocampal NMDA receptors and anxiety: at the interface between cognition and emotion. Eur J Pharmacol. 2010;626:49-56.

47. Krishnan-Sarin S, O'Malley SS, Franco N, et al. N-methyl-D-aspartate receptor antagonism has differential effects on alcohol craving and drinking in heavy drinkers. Alcohol Clin Exp Res. 2015;39:300-307.
48. Vengeliene V, Bachteler D, Danysz W, Spanagel R. The role of the NMDA receptor in alcohol relapse: a pharmacological mapping study using the alcohol deprivation effect. Neuropharmacology. 2005;48:822-829.

49. Xu J, Zhu Y, Kraniotis S, et al. Potentiating mGluR5 function with a positive allosteric modulator enhances adaptive learning. Learn Mem. 2013;20:438445.

50. Gass JT, Trantham-Davidson H, Kassab AS, Glen WB Jr, Olive MF, Chandler LJ. Enhancement of extinction learning attenuates ethanol-seeking behavior and alters plasticity in the prefrontal cortex. $J$ Neurosci. 2014;34:7562-7574.

51. Gass JT, Olive MF. Positive allosteric modulation of mGluR5 receptors facilitates extinction of a cocaine contextual memory. Biol Psychiatry. 2009;65:717-720.

52. Reid SD. Time for a regional alcohol policy: a literature review of the burden of normative alcohol use in the Caribbean. J Public Health Policy. 2015;36:469-483.

53. Love A, James D, Willner P. A comparison of two alcohol craving questionnaires. Addiction. 1998;93:1091-1102.

54. Chase HW, Eickhoff SB, Laird AR, Hogarth L. The neural basis of drug stimulus processing and craving: an activation likelihood estimation meta-analysis. Biol Psychiatry. 2011;70:785-793.

55. George O, Koob GF. Control of craving by the prefrontal cortex. Proc Natl Acad Sci USA. 2013;110:4165-4166.

56. Kumar J, Hapidin H, Bee Y-TG, Ismail Z. Effects of the mGluR5 antagonist MPEP on ethanol withdrawal induced anxiety-like syndrome in rats. Behav Brain Funct. 2013;9:43.

57. Bäckström P, Bachteler D, Koch S, Hyytia P, Spanagel R. mGluR5 antagonist MPEP reduces ethanol-seeking and relapse behavior. Neuropsychopharmacology. 2004;29:921-928.

58. Mihov Y, Hasler G. Negative allosteric modulators of metabotropic glutamate receptors subtype 5 in addiction: a therapeutic window. Int J Neuropsychopharmacol. 2016;19.

59. DeLorenzo C, DellaGioia N, Bloch M, et al. In vivo ketamine-induced changes in $\left[{ }^{11} \mathrm{C}\right] \mathrm{ABP} 688$ binding to metabotropic glutamate receptor subtype 5. Biol Psychiatry. 2015;77:266-275. 